\title{
A Novel Reduced-Size Rat-Race Broadside Coupler and Its Application for CMOS Distributed Sub-Harmonic Mixer
}

\author{
Chun-Lin Kuo, Che-Chung Kuo, Chun-Hsien Lien, Jeng-Han Tsai, Member, IEEE, and Huei Wang, Fellow, IEEE
}

\begin{abstract}
A broadband distributed sub-harmonic resistive field effect transistor mixer with a novel modified rat-race broadside coupler using a $0.13-\mu \mathrm{m}$ CMOS foundry process is presented in this letter. Using the broadside coupler, a reduced-size rat-race is fabricated with a compact size of $110 \times 80 \mu \mathrm{m}^{2}$. From the measurement results, the mixer achieved a conversion loss of $12 \mathrm{~dB}$ from 32 to $70 \mathrm{GHz}$ of RF frequency. The passive sub-harmonic mixer has zero power consumption. With the modified rat-race broadside coupler, the mixer achieves a good LO-IF isolation of better than $30 \mathrm{~dB}$ and a compact core chip size of $0.55 \times 0.4 \mathrm{~mm}^{2}$.
\end{abstract}

Index Terms-CMOS, distributed circuit, monolithic microwave integrated circuit (MMIC), sub-harmonic mixer.

\section{INTRODUCTION}

A sub-harmonic mixer allows the use of a local oscillator at a frequency that is only half of a fundamental mixer. Since the local oscillation (LO) frequency is relatively lower, the output power and phase noise can be superior to the fundamental source. The anti-parallel diode topology is most commonly used in passive sub-harmonic mixer design [1], [2]. However, the matching stubs using in the mixer restrain the bandwidth and the chip area. Another passive sub-harmonic mixer is the field effect transistor (FET) resistive mixer [3]. Active sub-harmonic mixers were proposed using the Gilbert-cell topology [4], but the requirement of a precise quadrature LO signal restrains the monolithic microwave integrated circuit (MMIC) design.

Recently, the bandwidth requirement is increasing in broadband and wireless communication system. As a good choice for high frequency application, distributed circuits are commonly used in the fundamental mixer design [5]-[7]. For a distributed sub-harmonic pumped mixer, the topology was only realized on hybrid circuit [8]. Distributed sub-harmonic mixers can achieve a wide bandwidth, but the bandwidth is usually restricted by the on-chip balun.

In this letter, we propose a broadband distributed sub-harmonic mixer with a reduced-size rat-race broadside coupler. The sub-harmonic mixer was fabricated using $0.13-\mu \mathrm{m}$ CMOS process. A novel reduced-size rat-race is implemented using

Manuscript received July 19, 2007; revised November 23, 2007. This work was supported by the National Science Council of Taiwan., R.O.C., under Projects NSC 93-2219-E-002-024, NSC 93-2213-E-002-033, and NSC93-2752-E-002-002-PAE and by TSMC, HsinChu, Taiwan, R.O.C., through the Chip Implementation Center, Hsinchu, Taiwan, R.O.C.

The authors are with the Department of Electrical Engineering and Graduate, Institute of Communication Engineering, National Taiwan University, Taipei 106, Taiwan, R.O.C. (e-mail: hueiwang@ew.ee.ntu.edu.tw).

Digital Object Identifier 10.1109/LMWC.2008.916806

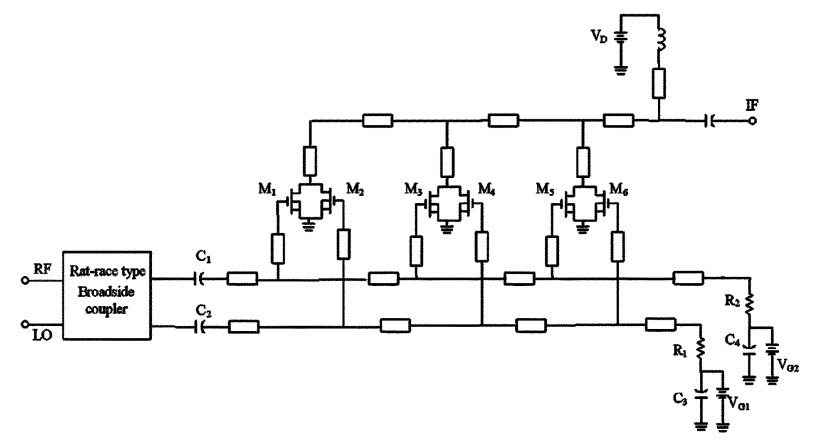

Fig. 1. Circuit schematic of the distributed sub-harmonic mixer with a rat-race type broadside coupler.

three metal layers forming a broadside coupler with a Marchand balun and two directional couplers. The measured results show that the conversion loss is $12 \mathrm{~dB}$ from 32 to $70 \mathrm{GHz}$ of RF frequency. The local oscillation intermediate frequency (LO-IF) isolation is better than $30 \mathrm{~dB}$ within the operation band. The output $P_{1 \mathrm{~dB}}$ is $-4 \mathrm{dBm}$ at $55 \mathrm{GHz}$ of RF frequency. The chip size is only $0.36 \mathrm{~mm}^{2}$ including bonding pads and the core area is only $0.22 \mathrm{~mm}^{2}$.

\section{Circuit Design And FABrication}

The schematic of the proposed distributed sub-harmonic mixer with a reduced-size, rat-race, broadside coupler is shown in Fig. 1. In the single mixer cell, a single-balanced topology is selected and the transistor pair, $M_{1}-M_{2}(48 \mu \mathrm{m} / 0.13 \mu \mathrm{m})$ are biased at nearly turn-off region. The LO and RF signals are applied to the gates of the transistor pair with differential and equal phase respectively.

Since the LO and RF signals are applied to the gates of the transistor pair, a rat-race hybrid is required in the design. However, a typical rat-race is fabricated using three $0.25 \lambda$ and one $0.75 \lambda$ transmission lines. It will occupy a lot of chip area. Thus, some modified rat-races such as those reported in [9]-[11] were used on chip for MMIC design, but the $0.25 \lambda$ transmission lines or lumped elements still occupy large chip areas. Conventional Marchand balun is a three-port element which can offer differential signals. In the letter, we proposed to add one more coupling element to extend the Marchand balun to a four-port modified rat-race with a miniature size. This concept can also be applied to a five-port dual balun for a different application (star mixer design) [12]. Here we design the broadside coupler with three metal layers. Top two layers (metal 8 and metal 7) is a three-port Marchand balun and we insert two lines (using metal 
(a)

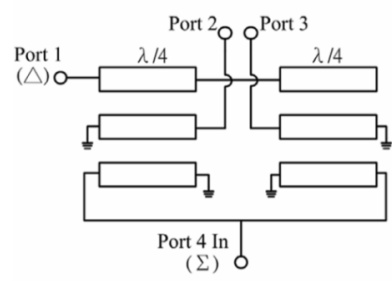

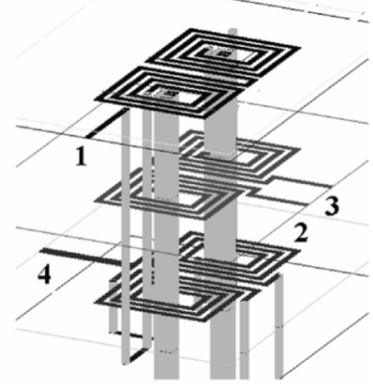

(b)
Fig. 2. The rat-race broadside coupler (a) structure (b) physical layout.

6) under the balun, as shown in Fig. 2(a). In operation frequency band, the signal of the port 1 will couple to the line of the next lower layer, and the port 2 and port 3 will be $180^{\circ}$ out of phase. In addition, the signal of the port 4 will couple to the line of next top layer, and the port 2 and port 3 will be in phase. Therefore, the LO signal from port 1 and RF signal from port 4 can be applied to the gates of the transistor pair with differential and equal phase, respectively. In order to design for low insertion loss, the low odd-mode impedance of the balun should be chosen [1], and the spacing and width of the couple-lines are determined accordingly. In addition, the length of the couple-lines is decided by the quarter wave length at the desired frequency, which is $800 \mu \mathrm{m}$ in this work. As shown in Fig. 2(b), these couple-lines are wound into a compact size of $110 \mu \mathrm{m} \times 80 \mu \mathrm{m}$, which is the same size of a Marchand balun. Finally, the full-wave EM simulator SONNET is used to determine the final dimensions of the rat-race broadside coupler. The line width and gap are both $3 \mu \mathrm{m}$ in this design.

The differential LO signals modulate the FET linear channel resistance and the mixing occurs due to the time-varying channel conductance. Three stages of mixer cell are selected with the trade-off between conversion gain and bandwidth. The transmission lines at gates are terminated to the characteristics impedance $\left(R_{1}=R_{2}=70 \Omega\right)$. At IF port, the desired IF signal frequency is usually lower than $1 \mathrm{GHz}$ thus it can be summed up by using short transmission lines at the drains and the termination resistor is also omitted at drain. The RF and twice of LO frequency leakages to the output port are far away from the IF and can be easily filtered out.

The broadside coupler and transmission lines are all complemented using thin-film microstrip (TFMS) structures with the bottom metal 1 as ground and top thick metal 8 as signals. All the passive components including the reduced-size rat-race broadside coupler, the MIM capacitors, and the TFMS lines were simulated using a full-wave EM simulator (Sonnet Software). The complete circuit was simulated using Agilent Advanced Design System (ADS).

\section{MEASURED RESUlTS}

The broadband distributed sub-harmonic mixer chip was measured using on-wafer probing. The output was connected to the spectrum analyzer through the bias-T network. The chip size is $0.6 \mathrm{~mm} \times 0.6 \mathrm{~mm}$, as show in Fig. 3. Fig. 4 shows the measured and simulated results of the reduced-size rat-race

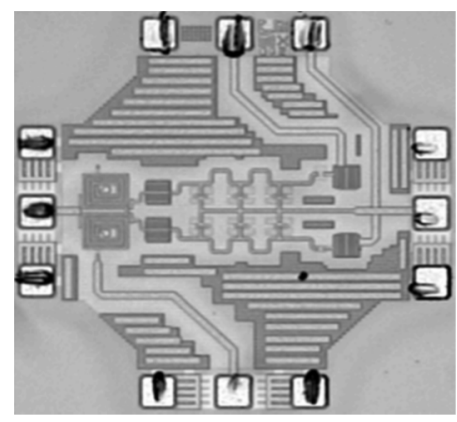

Fig. 3. Chip photograph of the distributed sub-harmonic mixer.

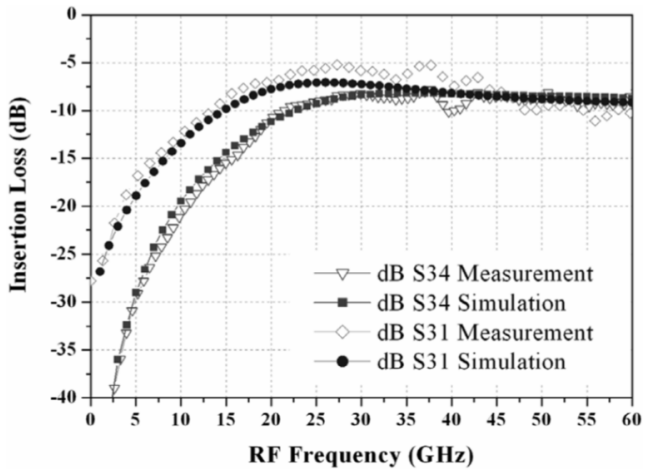

(a)

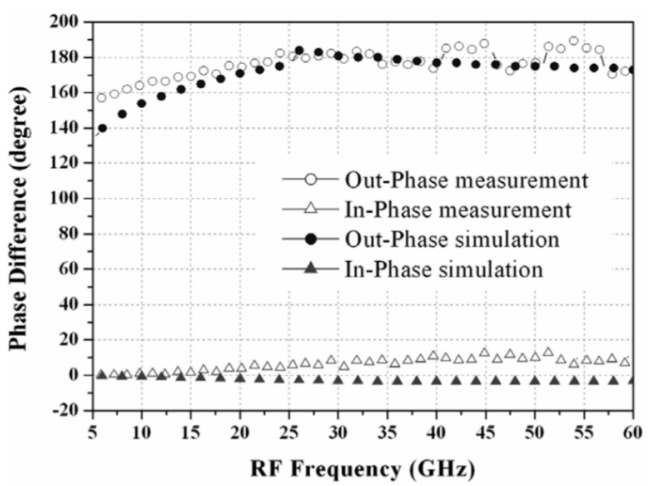

(b)

Fig. 4. Measured and simulated (a) insertion loss and (b) phase difference frequency response of the reduced-size rat-race broadside coupler test chip.

broadside coupler test circuit. The simulations agreed with the measurements reasonably. The measured insertion loss of the coupler is about $7 \mathrm{~dB}$; phase errors of in-phase and out-phase signals are within $8^{\circ}$ and $10^{\circ}$, respectively, from 30 to $60 \mathrm{GHz}$. The insertion loss which is about $4 \mathrm{~dB}$ higher than ideal case is due to the lossy Si substrate and the high resistivity of the thin metals using in the 2nd and 3rd layers of the balun coil.

The measured conversion loss swept with LO power is saturated at $13 \mathrm{dBm}$ at RF frequency of $55 \mathrm{GHz}$ and a fixed IF frequency of $1 \mathrm{GHz}$. Fig. 5 shows the measured conversion gain from 25 to $75 \mathrm{GHz}$. With the distributed topology, the circuit achieved a conversion loss of better than $12 \mathrm{~dB}$ from 32 to $70 \mathrm{GHz}$ with a fixed IF frequency of $1 \mathrm{GHz}$

Fig. 6 plots the measured port-to-port isolation. The LO-IF isolation is better than $30 \mathrm{~dB}$ due to the single-balanced topology and the 2LO-IF and LO-RF isolation is also better 
TABLE I

Summary of the Presently Published BRoAdBand Mixer AND This Work

\begin{tabular}{cccccc}
\hline Ref. & Process & Design Topology & $\begin{array}{c}\text { Bandwidth } \\
(\mathrm{GHz})\end{array}$ & Conversion Gain (dB) & $\begin{array}{c}\text { Area } \\
\left(\mathrm{mm}^{2}\right)\end{array}$ \\
\hline \hline This Work & $\begin{array}{c}0.13-\mu \mathrm{m} \\
\text { CMOS }\end{array}$ & $\begin{array}{c}\text { Subharmonic } \\
\text { Distributed }\end{array}$ & $32-70$ & $-11 \sim-13$ & 0.36 \\
\hline$[2]$ & $\begin{array}{c}0.15-\mu \mathrm{m} \\
\text { GaAs pHEMT }\end{array}$ & $\begin{array}{c}\text { Subharmonic } \\
\text { Anti-parallel Diode }\end{array}$ & $38-48$ & $-12 \sim-15$ & 0.72 \\
\hline$[13]$ & $\begin{array}{c}0.15-\mu \mathrm{m} \\
\text { GaAs pHEMT }\end{array}$ & $\begin{array}{c}\text { Subharmonic } \\
\text { Ring-quad }\end{array}$ & $25-40$ & -14 & 0.7 \\
\hline$[14]$ & $\begin{array}{c}90-\mathrm{nm} \\
\text { CMOS }\end{array}$ & $\begin{array}{c}\text { Subharmonic } \\
\text { Source-pumped }\end{array}$ & $9-31$ & $-8 \sim-11$ & 1 \\
\hline$[5]$ & $\begin{array}{c}0.15-\mu \mathrm{m} \\
\text { GaAs pHEMT }\end{array}$ & $\begin{array}{c}\text { Fundamental } \\
\text { Distributed }\end{array}$ & $3-33$ & $-1 \sim-4$ & 1.7 \\
\hline$[7]$ & $\begin{array}{c}0.15-\mu \mathrm{m} \\
\text { GaAs pHEMT }\end{array}$ & $\begin{array}{c}\text { Fundamental } \\
\text { Distributed }\end{array}$ & $4-41$ & $3.5-8$ & 2 \\
\hline
\end{tabular}

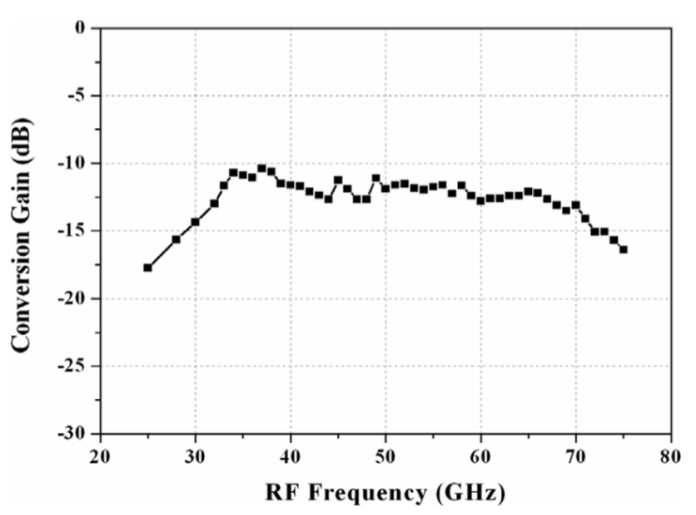

Fig. 5. Measured conversion gain versus RF frequency with a LO power of 13 $\mathrm{dBm}$ and a fixed IF frequency of $1 \mathrm{GHz}$.

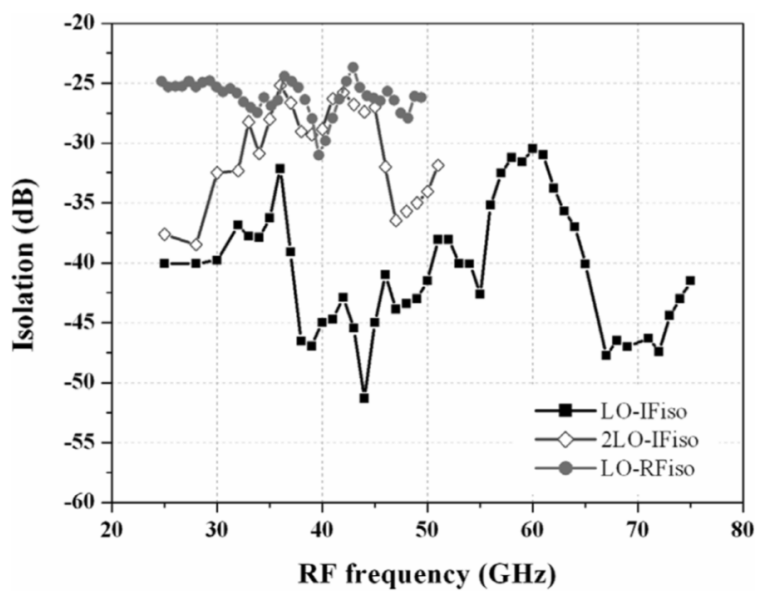

Fig. 6. Measured port-to-port isolation.

than $20 \mathrm{~dB}$. The output $1-\mathrm{dB}$ compression point of the distributed sub-harmonic mixer is about $-4 \mathrm{dBm}$ at RF of $55 \mathrm{GHz}$. Table I summarizes the performance of previously published wide-band sub-harmonic mixer integrated-circuit [13], [14]. The MMIC in this letter is believed to be the widest bandwidth CMOS sub-harmonic mixer to date.

\section{CONCLUSION}

A broadband 32 to $70 \mathrm{GHz}$ distributed sub-harmonic mixer is designed and fabricated using TSMC $0.13-\mu \mathrm{m}$ CMOS process. A novel reduced-size rat-race using broadside coupler is proposed to provide an out-phase LO signal and an in-phase RF signal simultaneously. With the broadside coupler, the distributed sub-harmonic mixer achieves a broad bandwidth with a compact size. The sub-harmonic mixer exhibits $-12 \pm 1 \mathrm{~dB}$ measured conversion gain from 32 to $70 \mathrm{GHz}$ with a miniature chip size of $0.36 \mathrm{~mm}^{2}$.

\section{REFERENCES}

[1] S. A. Mass, Microwave Mixers. Norwell, MA: Artech House, 1993.

[2] W.-C. Chen, S.-Y. Chen, J.-H. Tsai, T.-W. Huang, and H. Wang, "A 38-48-GHz miniature MMIC subharmonic mixer," in Proc. Gallium Arsenide Appl. Symp. (GAAS'05), Oct. 2005, pp. 437-440.

[3] M.-F. Lei, P.-S. Wu, T.-W. Huang, and H. Wang, "Design and analysis of a miniature W-Band MMIC subharmonically pumped resistive mixer," in IEEE MTT-S Int. Dig., Jun. 2004, pp. 235-238.

[4] L. Sheng, J. C. Jensen, and L. E. Larson, "A wide-bandwidth Si/SiGe HBT direct conversion sub-harmonic mixer/downconverter," IEEE J. Solid-State Circuits, vol. 35, no. 9, pp. 1329-1337, Sep. 2000.

[5] K.-L. Deng and H. Wang, "A 3-33 GHz pHEMT MMIC distributed drain mixer," in IEEE RFIC Symp. Dig., Jun. 2002, pp. 151-154.

[6] W. Ko and Y. Kwon, "A GaAs-based 3-40 GHz distributed mixer with cascade FET cells," in RFIC Symp. Dig., Jun. 2004, pp. 413-416.

[7] F.-C. Chang, P.-S. Wu, M.-F. Lei, and H. Wang, "A 4-41 GHz singly balanced distributed mixer using GaAs pHEMT technology," IEEE Microw. Wireless Compon. Lett., vol. 17, no. 2, pp. 136-138, Feb. 2007.

[8] A. H. Darsinooieh and O. Palamutcuoglu, "On the theory and design of subharmonically drain pumped microwave MESFET distributed mixers," in Proc. Electrotech. Conf., 1996, vol. 1, pp. 595-598.

[9] P. Phillipe and M. Pertus, "A 2-GHz enhancement mode GaAs down converter IC for satellite TV tuner," in IEEE Microw. Millimeter-Wave Monolith. Circuits Symp. Dig., 1991, pp. 61-61.

[10] T. Hirota, A. Minakawa, and M. Muraguchi, "Reduced-size branchline and rat-race hybrid for uniplanar MMIC's," IEEE Trans. Microw. Theory Tech., vol. 38, no. 3, pp. 270-275, Mar. 1990.

[11] K. S. Ang and Y. C. Leong, "Converting baluns into broad-band impedance-transforming $180^{\circ}$ hybrids," IEEE Trans. Microw. Theory Tech., vol. 50, no. 8, pp. 1990-1995, Aug. 2002.

[12] C.-C. Kuo, C.-L. Kuo, C.-J. Kuo, S. A. Mass, and H. Wang, "Novel miniature and broad-band millimeter-wave monolithic star mixers," IEEE Trans. Microw. Theory Tech., vol. 56, no. 4, Apr. 2008, to be published.

[13] P. Blount and C. Trantanella, "A high IP3, subharmonically pumped mixer for LMDS application," in Proc. Gallium Arsenide Appl. Symp. (GAAS'00), Nov. 2000, pp. 171-174.

[14] M. Bao, H. Jacobsson, L. Aspemyr, G. Carchon, and X. Sun, "A 9-31-GHz subharmonic passive mixer in 90-nm CMOS technology," IEEE J. Solid-State Circuits, vol. 11, no. 10, pp. 2257-2264, Oct. 2006. 